\title{
Clinical efficacy of ozagrel with or without edaravone in 156 acute stroke patients
}

\author{
Shun Aritake, Hiroaki Oguro*, Kenichi Iwasa, Shingo Mitaki, Hiroyuki Takayoshi, Satoshi Abe, Keiichi Onoda, \\ Shuhei Yamaguchi \\ Department of Neurology, Shimane University Hospital, 89-1 Enya-cho, Izumo city, Shimane 693-8501, Japan
}

\section{Article Info}

Article Notes

Received: May 22, 2017

Accepted: June 12, 2017

\section{*Correspondence:}

Hiroaki Oguro, MD

Department of Neurology, Shimane University Hospital, 89-1

Enya-cho, Izumo city, Shimane 693-8501, Japan

Email: oguro@med.shimane-u.ac.jp

(c) 2017 Oguro $\mathrm{H}$. This article is distributed under the terms of the Creative Commons Attribution 4.0 International License

\section{Keywords}

Ozagrel

Edaravone

Stroke

JSSRS

Atherothrombotic infarction

Lacunar infarction

\section{ABSTRACT}

We studied prognosis in 156 acute stoke patients treated by ozagrel and edaravone with the Japanese Standard Stroke Registry database in our hospital. They were examined as to their stroke types, neurological severity according to the NIH Stroke Scale (NIHSS) and clinical outcomes at admission by the modified Rankin Scale (mRS). Acute noncardioembolic stroke patients with lacunar and atherothrombosis showed functional recovery by both of ozagrel monotherapy and the combination therapy of ozagrel and edaravone. Although ozagrel monotherapy in atherothrombotic infarction showed most improved by 2.4 points, we did not find significant difference among four groups according to ozagrel monotherapy or the combination therapy in atherothrombotic or lacunar infarct after adjusted for age.

\section{Introduction}

Sodium ozagrel (Ozagrel ${ }^{\circledR}$ ) is a thromboxane A2 inhibitor agent for applicable for acute noncardioembolic ischemic stroke in Japan from 1992. However, there is not so many studies evaluating its effect on acute ischemic stroke. We have studied prognosis of acute ischemic stroke patients with the Japanese Standard Stroke Registry Study (JSSRS) database in our hospital. They were examined as to their stroke types, their severity of stroke according to the NIH Stroke Scale (NIHSS) and outcomes by the modified Rankin Scale (m-RS). During period from January 2001 to December 2016, we treated 656 patients with acute cerebral infarction by ozagrel. Retrospectively we evaluated the efficacy of ozagrel by using NIHSS and modified Rankin Scale (mRS). We frequently add edaravone, free radical scavenger to ozagrel for the treatment of acute ischemic stroke. We evaluated the additional effect of edaravone to ozagrel, and we made comparison between ozagrel monotherapy and combination of ozagrel and edaravone.

\section{Subjects and Method}

We enrolled 156 acute stroke patients who had suffered atherothrombotic infarction (ATI) or lacunar infarction (LI) from the Japanese Standard Stroke Registry Study (JSSRS) database ${ }^{1-3}$. They were treated either with ozagrel monotherapy (99 patients; LI: 76, ATI: 23) or with combination of ozagrel and edaravone (57 patients; LI: 35, ATI: 22) within 48 hours from onset and registered in JSSRS ${ }^{4}$. Chronic kidney disease and hemodialysis cases were excluded in this study, because we have difficulty in using edaravone for renal dysfunction. Ozagrel (80mg) was administered intravenously by drip infusion over $2 \mathrm{~h}$ b.i.d, in the morning and the evening. Edaravone (30mg) was 
administered intravenously by drip infusion over 30 min b.i.d, in the morning and the evening. Concomitant use of argatroban, urokinase, heparin, warfarin, aspirin, ticlopidine hydrochloride, clopidogrel, and cilostazol were prohibited during the administration period of ozagrel and edaravone. The modified Rankin Scale (mRS) and National Institute of Health Stroke Scale (NIHSS) are used for assessment of clinical outcome on admission and discharge ${ }^{5}$. We evaluated the efficacy of ozagrel monotherapy or combination therapy with edaravone and ozagrel. The improvement of NIHSS with more than 2 points between admission and discharge are classified as good outcome on discharge. This JSSRS database study was approved by Shimane University Institutional Committee on Ethics (registered in 2001).

\section{Statistical analysis}

The chi-square test was used to analyze differences in clinical factors including gender, stroke subtype and concurrent antiplatelet between pre/post therapy of ozagrel and with/without edaravone. The Wilcoxon signed rank sum test was used to analyze the NIHSS scores and mRS between pre/post therapy of ozagrel and with/ without edaravone. Logistic regression analysis was used to determine the relationship between good outcome at discharge and clinical factors including NIHSS and mRS at admission, age, gender, blood pressure on admission, time to treatment after stroke onset, concurrent antiplatelet, stroke subtype (LI or ATI) and therapeutic groups (monotherapy or combination therapy). P values of $<0.05$ were considered significant. All values are presented as means \pm standard deviations.

\section{Results}

156 acute noncardioembolic ischemic stroke patients were enrolled retrospectively from JSSRS database. Significant intergroup difference in baseline characteristics was only observed for age $(\mathrm{p}<0.05)$. Of stroke patients, 99 cases were administered with ozagrel including 76 LI cases $(76.7 \%)$ and 23 ATI cases (23.3\%). Other 57 cases including $35 \mathrm{LI}$ and 22 ATI were administered with the combination therapy, edaravone and ozagrel.
This distribution about stroke subtype and concurrent antiplatelet were not significant between monotherapy and combination therapy (Table 1). Of 156 all patients, mild stroke cases with NIHSS less than 6 point were found in 128 cases $(82 \%)$ including 96 cases in 111 LI patients (86\%) and 32 cases in 45 ATI group (71\%). In LI group, ozagrel monotherapy with 76 cases showed NIHSS were improved by -1.4 (pre $=3.6$, post $=2.2, \mathrm{p}<0.0001$ ). 35 cases with combination therapy of edaravone and ozagrel showed NIHSS were improved by -1.2 (pre $=2.9$, post $=1.7$, $\mathrm{p}<0.001$ ). There is not difference in NIHSS improvement between ozagrel monotherapy and combination therapy in LI group. In ATI group, ozagrel monotherapy with 23 cases showed NIHSS were improved by -2.4 (pre $=4.4$, post $=2.0$, p $<0.001) .22$ ATI cases with combination therapy were not significantly improved by -0.8 (pre $=4.0$, post $=3.2$ ). Ozagrel monotherapy had revealed clinical effectiveness in both of LI $(\mathrm{p}<0.0001)$ and ATI $(\mathrm{p}<0.001)$.

The NIHSS improvement (-2.4) by ozagrel monotherapy in 23 cases of ATI group was larger than other three groups (both therapy in LI group and combination therapy in ATI), but we did not find statistical difference among four groups by evaluating analysis of covariance after adjusted for age (Table 2). The ratio of mild symptom (m-RS 0-1) in 99 cases treated by ozagrel monotherapy had significantly increased from 15 patients on admission (15\%) to 45 patients on discharge $(45 \%, \mathrm{p}<0.0001)$ (Figure A). The ratio of mild symptom (m-RS 0-1) in 57 treated by combination therapy had significantly increased from 5 patients on admission (9\%) to 28 patients on discharge $(49 \%, \mathrm{p}<0.001)$ (Figure B). Logistic regression analysis demonstrated that a good outcome was only related to the NIHSS at admission (OR, 0.675; 95\% CI, 0.551- 0.827, $\mathrm{p}<0.001$ ). Other clinical factors, age, gender, blood pressure on admission, time to treatment after stroke onset, concurrent antiplatelet, stroke subtype (LI or ATI) and therapeutic groups (monotherapy or combination therapy) are all not related with good outcome.

\section{Discussion}

To investigate the state of ischemic stroke treatment in

\begin{tabular}{|c|c|c|c|}
\hline & Monotherapy of ozagrel $n=99$ & Combination therapy of ozagrel and edaravone $n=57$ & p value \\
\hline Age & $71.5 \pm 10.4$ & $75.5 \pm 11.2$ & 0.0169 \\
\hline Gender (M/F) & $63 / 36$ & $39 / 18$ & 0.6671 \\
\hline Time to treatment after stroke onset & $20.4 \pm 17.4$ & $16.3 \pm 11.8$ & 0.3144 \\
\hline \multicolumn{4}{|l|}{ Blood pressure on admission } \\
\hline Systolic blood pressure & $155.1 \pm 28.0$ & $161.1 \pm 24.0$ & 0.1185 \\
\hline Diastolic blood pressure & $86.2 \pm 15.9$ & $86.2 \pm 14.3$ & 0.8871 \\
\hline \multicolumn{4}{|l|}{ Stroke subtype } \\
\hline Lacunar infarction (LI) & 76 & 35 & \multirow{2}{*}{0.0634} \\
\hline Atherothrombotic infarction (ATI) & 23 & 22 & \\
\hline Concurrent antiplatelet (+/-) & $17 / 82$ & $12 / 45$ & 0.6993 \\
\hline
\end{tabular}

Table 1: Baseline characteristics 


\begin{tabular}{|c|c|c|c|c|}
\hline & \multicolumn{2}{|c|}{ Lacunar Infarction (LI) group } & \multicolumn{2}{|c|}{ Atherothrombotic Infarction (ATI) group } \\
\hline & Ozagrel monotherapy & $\begin{array}{c}\text { Combination with ozagrel } \\
\text { and edaravone }\end{array}$ & Ozagrel monotherapy & $\begin{array}{c}\text { Combination with ozagrel } \\
\text { and edaravone }\end{array}$ \\
\hline $\mathrm{n}$ & 76 & 35 & 23 & 22 \\
\hline NIHSS at admission & $3.6(2.7)$ & $2.9(2.1)$ & $4.4(3.4)$ & $4.0(3.2)$ \\
\hline NIHSS at discharge & $2.2(2.7)$ & $1.7(1.9)$ & $2.0(3.1)$ & $3.2(3.0)$ \\
\hline improvement of NIHSS & $-1.4(1.7)$ & $-1.2(1.4)$ & $-2.4(2.0)$ & $-0.8(2.2)$ \\
\hline mRS at admission & $2.8(1.3)$ & $2.7(1.2)$ & $3.0(1.3)$ & $3.0(1.2)$ \\
\hline $\mathrm{mRS}$ at discharge & $1.9(1.5)$ & $1.6(1.3)$ & $1.9(1.5)$ & $2.4(1.5)$ \\
\hline
\end{tabular}

Table 2 : Clinical outcomes in subtype of stroke and therapy.

A

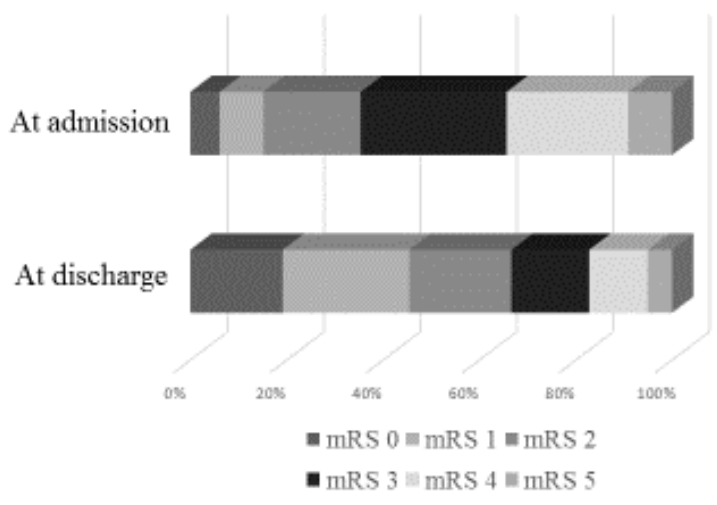

$\mathrm{p}<0.0001$
B

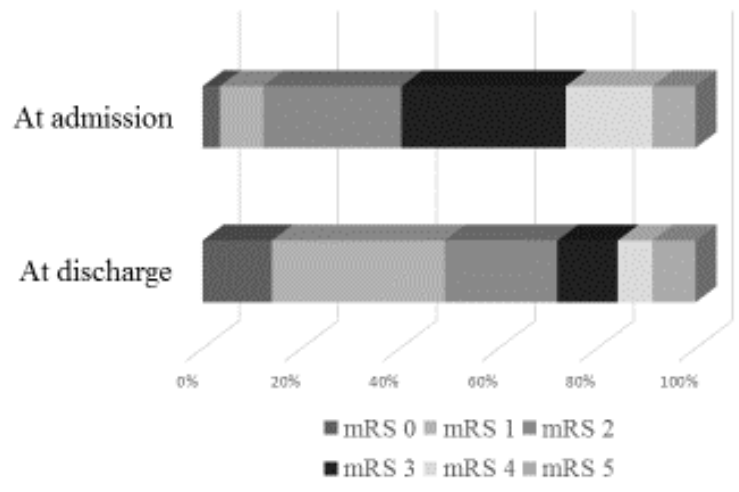

$\mathrm{p}<0.001$

Figure: Distribution of modified Rankin scale score on admission and discharge.

A. Ozagrel monotherapy.

B. Combination therapy by ozagrel and edaravone.

Japan, many Japanese stroke neurologists analyzed data from stroke patients registered in the Japanese Standard Stroke Registry Study (JSSRS) database within the last seventeen years ${ }^{2,3}$. Our laboratory analyzed the amassed data of JSSRS about all japan with 9197 stroke cases in 2005 . Sodium ozagrel was the drug used most frequently to treat patients with LI (75\%), so it equals to our laboratory's data in this study ${ }^{3}$. Wada et al reported that ozagrel for atherothrombotic stroke reduced readmission for stroke recurrence within 90 days, but ozagrel monotherapy was not associated with mRS at discharge in both of LI and ATI by propensity score matched and retrospective analysis ${ }^{6}$.

In acute ischemic stroke, platelets are activated with releasing neurotoxic and thrombo-genic eicosanoids which could cause brain damage secondary to reducing the brain blood flow. Ozagrel, a thromboxane A2 synthase inhibitor decrease platelet aggregation and increase cerebral blood flow by decreasing thromboxane A2 which has a plateletaggregating and a vasoconstrictive action ${ }^{7,8}$. Ozagrel reduces the risk of neurological impairment and the death for acute ischemic stroke, so it is frequently used to treat ischemic cerebral thrombosis 9 . The Japanese Guidelines for the Management of Stroke 2015 suggest ozagrel use as a grade B recommendation in acute noncardioembolic ischemic stroke within five days from onse ${ }^{10}$. Ozagrel was approved for motor weakness caused by noncardioembolic ischemic stroke by Japanese Ministry of Health and Welfare in 1992. Mild stroke patients had tendency to treat with edaravone, ozagrel and the combination of two drugs. Although the adverse events of ozagrel were major bleeding events including digestive hemorrhage or hemorrhagic stroke, we had not any major bleeding events. In our study, ozagrel had revealed clinical effectiveness in both of LI group $(p<0.0001)$ and ATI group $(p<0.001)$ whether we added edaravone or not. During scheduled treatment, ozagrel is effective for the improvement of neurological impairment for stroke patients.

Edaravone has been reported to inhibit tissue injury 
including vascular endothelial cell, delayed neuronal death with inhibition of peroxidation of the phosphatidylcholine liposomal membrane initiated by free radicals and antioxidants, ascorbic acid and $\alpha$-tocopherol ${ }^{11,12}$. Ozawa et al reported that there is no difference in neurological status between 27 cases with ozagrel monotherapy and 27 cases with combination of ozagrel and edaravone. They assumed that mild stroke cases do not have apparent effectiveness in edaravone therapy because edaravone has a clinical effect on moderate cases with atheromatous infarction ${ }^{13}$. Yamaguchi et al reported edaravone have clinically beneficial interactions with ozagrel regarding the prevention of vascular atherosclerosis by animal experiment revealing antiproliferative effect of the basilar artery smooth muscle cells from guinea-pig ${ }^{14}$. In our study, we could not find combination therapy of ozagrel and edaravone was not superior to ozagrel monotherapy in clinical outcome. Edaravone is equally as effective as ozagrel in ischemic stroke, but combination therapy of edaravone and ozagrel could not be superior to ozagrel monotherapy.

There were several limitations of our study. Our study is only cross sectional analysis, so it would not be clear in prognosis of noncardioembolic ischemic stroke patients treated by ozagrel and edaravone. We need to continue the prospective study about clinical outcome of noncardioembolic ischemic stroke treatment by ozagrel and edaravone in JSSRS database.

\section{Conclusion}

Both Ozagrel monotherapy and combination therapy of ozagrel with edaravone are effective on acute noncardioembolic ischemic stroke. Low score of NIHSS at admission would be a good outcome in prognosis.

\section{References}

1. Bokura H, Kobayashi S, Yamaguchi S, et al. Clinical characteristics and prognosis in stroke patients with diabetes mellitus: retrospective evaluation using the Japanese Standard Stroke Registry database (JSSRS). No To Shinkei. 2006; 58(2): 135-139.

2. Kobayashi S, Nakagawara J, Ogawa A. Clinical evaluation of Japan Stroke Scale compared with NIH Stroke Scale in acute ischemic stroke patients: from Japanese stroke data bank. Stroke. 2003; 34: 317.

3. Okada K, Kobayashi S. The present state of acute ischemic stroke therapy in Japan. Internal Medicine. 2005; 44(4): 365-368.

4. Okada K, Kobayashi S, JSSRS Group. Therapeutic strategy for acute stroke prologue for an epoch of brain attack. The present state of acute ischemic stroke therapy in Japan. Intern Med. 2005; 44(4): 365-368.

5. van Swieten JC, Koudstaal PJ, Visser MC, et al. Interobserver agreement for the assessment of handicap in stroke patients. Stroke. $1988 ; 19(5): 604-607$.

6. Wada T, Yasunaga $\mathrm{H}$, Horiguchi $\mathrm{H}$, et al. Ozagrel for Patients With Noncardioembolic Ischemic Stroke: A Propensity Score-Matched Analysis. J Stroke Cerebrovasc Dis. 2016; 25(12): 2828-2837.

7. Oishi M, Mochizuki Y, Hara M, et al. Effects of sodium ozagrel on hemostatic markers and cerebral blood flow in lacuna infarction. Clin Neuropharrnacol. 1996; 19: 526-531.

8. Nihei C, Metoki H, Shimanaka Y, et al. Clinical study on effect of thromboxane A2 synthetase inhibitor (OKY-046)to clarify the regulation of cerebral blood flow in cerebral thrombosis. Adv Prostaglandin Thromboxane Leukotriene Res. 1987; 17B: 953-957.

9. Zhang J, Yang J, Chang X, et al. Ozagrel for acute ischemic stroke: a meta-analysis of data from randomized controlled trials. Neurol Res. 2012; 34(4): 346-353.

10. The Joint Committee on Guidelines for the Management of Stroke: Japanese Guidelines for the Management of Stroke. 2015.

11. Group EAIS. Effect of a novel free radical scavenger, edaravone (MCI186) on acute brain infarction Randomized placebo-controlled double blind study at multicenters. Cerebrovasc Dis. 2003; 15(3): 222-229.

12. Yamamoto $\mathrm{Y}$, Kuwahara T, Watanabe $\mathrm{K}$, et al. Antioxidant activity of 3-methyl-1-phenyl-2-pyrazolin-5-one. Redox Rep. 1996; 2(5): 333-338.

13. Ozawa T, Fujii Y, Tanaka R. Evaluation of the effect of combination therapy with edaravone and sodium ozagrel on acute ischemic stroke. Jpn J Stroke. 2005; 27: 283-291.

14. Yamaguchi T, Oishi K, Uchida $\mathrm{M}$, et al. Edaravone, a radical scavenger may enhance or produce antiproliferative effects of fluvastatin, amlodipine, ozagrel, GF109203X and Y27632 on cultured basilar artery smooth muscle cells. Biol Pharm Bull. 2003; 26(12): 17061710. 